\title{
Slow dynamics and subdiffusion in a non-Hamiltonian system with long-range forces
}

\author{
Romain Bachelard, ${ }^{1, *}$ Nicola Piovella, ${ }^{2, \dagger}$ and Shamik Gupta ${ }^{3, \ddagger}$ \\ ${ }^{1}$ Departamento de Fisica, Universidade Federal de São Carlos, Rodovia Washington Luis, km 235, S/n Jardim Guanabara, São Carlos, \\ São Paulo 13565-905, Brazil \\ ${ }_{2}^{2}$ Dipartimento di Fisica Aldo Pontremoli, Università degli Studi di Milano, via G. Celoria 16, 20133 Milano, Italy \\ ${ }^{3}$ Department of Physics, Ramakrishna Mission Vivekananda University, Belur Math, Howrah 711202, India
}

(Received 24 July 2018; published 18 January 2019)

\begin{abstract}
Inspired by one-dimensional light-particle systems, the dynamics of a non-Hamiltonian system with longrange forces is investigated. While the molecular dynamics does not reach an equilibrium state, it may be approximated in the thermodynamic limit by a Vlasov equation that does possess stable stationary solutions. This implies that on a macroscopic scale the molecular dynamics evolves on a slow timescale that diverges with the system size. At the single-particle level, the evolution is driven by incoherent interaction between the particles, which may be effectively modeled by a noise, leading to a Brownian-like dynamics of the momentum. Because this self-generated diffusion process depends on the particle distribution, the associated Fokker-Planck equation is nonlinear, and a subdiffusive behavior of the momentum fluctuations emerges, in agreement with numerics.
\end{abstract}

DOI: 10.1103/PhysRevE.99.010104

Long-range interactions are present at all scales, from atomic physics to astrophysics, from hydrodynamics to plasma and free-electron laser physics [1]. The lack of additivity of long-range systems challenges several important results of equilibrium statistical physics found in classical textbooks and developed for short-range interactions. The most fundamental consequences are the possibility of a nonconcave entropy [2] and inequivalent microcanonical and canonical ensembles [3].

It is probably when out of equilibrium that long-range systems revealed most surprises, with the rather intriguing and interesting property that the time to reach equilibrium may diverge with the system size [4,5]. Coined "quasistationarity," this peculiar behavior was shown to derive from the existence of the so-called Vlasov equation describing the phasespace dynamics in the thermodynamic limit, which admits a continuum of stable stationary solutions [6]. An important consequence is that large systems may essentially remain trapped in out-of-equilibrium states for times accessible to experiments. These results, obtained for energy-conserving Hamiltonian dynamics, were nevertheless contrasted by studies of dynamics that violates energy conservation, in which stochastic terms were shown to put a bound on the lifetimes of the out-of-equilibrium states [7-9]. Nevertheless, until now, the Hamiltonian dynamics has been the main framework to study the phenomena of quasistationarity, as an heritage of statistical physics.

In this Rapid Communication, we show that nonHamiltonian systems with long-range forces may also exhibit quasistationary features, despite not ever reaching an equilibrium. The model under consideration, which may be

\footnotetext{
*bachelard.romain@gmail.com

†nicola.piovella@mi.infn.it

†shamik.gupta@rkmvu.ac.in
}

achieved either in cold atom or free-electron laser setups, has an ever-growing kinetic energy. We show that the existence of a general condition for the stability of stationary solutions of the associated Vlasov equation allows for the presence of quasistationary states. For nonmagnetized states, each particle is driven by a fluctuating magnetization that can effectively be modeled as a stochastic noise, which in turn allows us to derive a nonlinear Fokker-Planck equation for the momentum distribution. Assuming that the system reaches a Gaussian distribution in momentum, a subdiffusive behavior of momentum fluctuations is predicted, in agreement with our numerical findings. Our work reveals a surprising dynamical possibility allowed by non-Hamiltonian long-range forces. Thermodynamically, the system does not have a long-time equilibrium stationary state to relax to. Nevertheless, dynamically, the system remains trapped in states for times that diverge with the system size, so that such states become in the limit of large system size the effective stationary states of the system. This work is to the best of our knowledge the first demonstration of quasistationarity in non-Hamiltonian long-range systems. We also offer possible experimental platforms to observe our predicted findings.

The physical model we consider here is the onedimensional dynamics of particles interacting with light, as may be achieved in free-electron laser [10] and cold atom [11] setups. In these systems, the particles typically behave as pendula coupled by the common radiation field. For example, a cloud of cold atoms in a ring optical cavity backscatters the photons from an incident pump beam into a counterpropagating cavity mode, according to the following equations:

$$
\begin{aligned}
& \dot{\theta}_{j}=p_{j}, \quad \dot{p}_{j}=-g\left(A e^{i \theta_{j}}+\text { c.c. }\right), \\
& \dot{A}=\frac{g}{N} \sum_{j=1}^{N} e^{-i \theta_{j}}-(\kappa-i \Delta) A,
\end{aligned}
$$


where $\theta_{j}, p_{j}$, and $A \propto 1 / \sqrt{N}$ are respectively the normalized positions and the momenta of the $N$ particles and the cavity field amplitude, while c.c. stands for complex conjugate. Here, $g \propto \sqrt{N}$ describes the coupling between the atoms and the field $[10,11], \kappa$ models the cavity losses, and $\Delta$ is the frequency mismatch between the cavity and the atomic transition. The $1 / N$ term in Eq. (1b) allows consideration of the thermodynamic limit $(N \rightarrow \infty)$ of the problem without encountering divergences, in accordance with the Kac prescription [12].

For a bad-quality cavity $\left(\kappa>g^{2 / 3}\right)$, the scattered field quickly leaves the interaction region, while the atoms continuously lose momentum by emitting photons into the cavity mode. The system then enters into a superradiant regime in which the atoms scatter a transient radiation pulse with intensity proportional to $N^{2}$. The same regime can be achieved in a free-electron laser operating with short electron bunches [13]. The adiabatic elimination of the field amplitude reads $A \approx g /(\kappa-i \Delta) \sum_{j=1}^{N} e^{-i \theta_{j}} / N$, which in turn leads to the following equations:

$$
\begin{aligned}
\dot{\theta}_{j}= & p_{j}, \\
\dot{p}_{j}= & -\frac{2 g^{2} \kappa}{\kappa^{2}+\Delta^{2}} \frac{1}{N} \sum_{m=1}^{N} \cos \left(\theta_{j}-\theta_{m}\right) \\
& +\frac{2 g^{2} \Delta}{\kappa^{2}+\Delta^{2}} \frac{1}{N} \sum_{m=1}^{N} \sin \left(\theta_{j}-\theta_{m}\right) .
\end{aligned}
$$

For far-detuned light $(|\Delta| \gg \kappa)$, the cosine term in the second equation may be dropped, and one recovers a Hamiltonian dynamics that has been studied extensively under the name of the Hamiltonian mean-field model [14]. On the contrary, at resonance $(\Delta=0)$, the dynamics is strongly dissipative, a case on which we focus from now on. Also, since it corresponds to a rescaling of time and momentum, we set from now on $2 g^{2} \kappa /\left(\kappa^{2}+\Delta^{2}\right)=1$ without loss of generality.

The macroscopic ordering of the particles is captured by the magnetization $M \equiv(1 / N) \sum_{j=1}^{N} e^{-i \theta_{j}}$ that may be used to rewrite the dynamical equations as

$$
\dot{\theta}_{j}=p_{j}, \dot{p}_{j}=-\frac{1}{2}\left(M e^{i \theta_{j}}+M^{*} e^{-i \theta_{j}}\right) .
$$

An important feature is that the force $F_{j m}=$ $-(1 / N) \cos \left(\theta_{j}-\theta_{m}\right)$ on particle $m$ due to particle $j$ does not have the symmetry of a force derivable from a two-body interaction potential that is a function solely of the separation between particles. In the latter case, one has $\mathbf{F}_{j m}=-\mathbf{F}_{m j}$, which is the situation typical of Hamiltonian systems encountered in statistical mechanics, and which ensures that the value of the average momentum $P \equiv(1 / N) \sum_{j=1}^{N} p_{j}$ is conserved in time. The dynamics (3) is not derivable from an underlying Hamiltonian, so that one may not associate an energy function with the system. The average momentum for our model is not conserved but instead decreases in time according to

$$
\dot{P}=-|M(t)|^{2} .
$$

Even in a nonmagnetized phase, while $M(t)$ averages to zero over time, the fluctuations of $|M|$ will contribute to the decrease of the total momentum. Consequently, the system

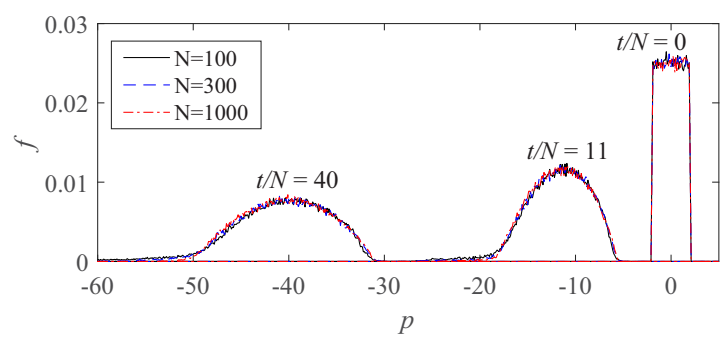

FIG. 1. The single-particle $p$ distribution as a function of rescaled time $t / N$ for the dynamics (3) for three different systems size $N$ and for an initial state that is WB with $\Delta p=0.5$. The data are obtained from numerical integration of the dynamics (3) for different system sizes.

does not possess a proper equilibrium, with a momentum distribution that is stationary in time.

The decrease of $P$ with time is confirmed by numerical simulations of the dynamics (3), as may be concluded from Fig. 1 by observing the shift of the center of the momentum distribution and the collapse of the curves for different system size $N$ on scaling time by $N$. The latter observation implies a rather strong dependence of the dynamics on the system size $N$, suggesting a slowing down of the evolution with increase of $N$. Similar slowdown of macroscopic evolution in systems with long-range interaction has already been reported for Hamiltonian dynamics [1,15], and may be explained as resulting from the occurrence of a continuum of stable stationary solutions of the Vlasov equation describing the macroscopic evolution of the system in the thermodynamic limit $[1,16]$.

Although our model (3) is intrinsically non-Hamiltonian, it is instructive, especially in the light of our observation of slow relaxation mentioned above, to derive a Vlasov equation to describe its dynamics in the limit of large $N$. To this end, let us introduce the single-particle density $f_{d}(\theta, p, t) \equiv$ $(1 / N) \sum_{j=1}^{N} \delta\left(\theta-\theta_{j}(t)\right) \delta\left(p-p_{j}(t)\right)$ as the density of particles with angle $\theta$ and momentum $p$ at time $t$. Taking the time derivative of $f_{d}$ and using the equations of motion (3), it may be shown that in the limit of large $N$, when the discrete function $f_{d}(\theta, p, t)$ approaches a continuous one, namely, the single-particle distribution function $f(\theta, p, t)$, the time evolution of the latter is given by a Vlasov equation of the following form (for the general procedure, see Ref. [1]):

$$
\frac{\partial f}{\partial t}+p \frac{\partial f}{\partial \theta}+F[f](\theta, t) \frac{\partial f}{\partial p}=0 .
$$

Here, $\quad F[f](\theta, t) \equiv-\iint d \theta^{\prime} d p^{\prime} f\left(\theta^{\prime}, p^{\prime}, t\right) \cos \left(\theta-\theta^{\prime}\right), \quad$ a functional of $f$, is the net force experienced by a particle with angle $\theta$ at time $t ; f(\theta, p, t)$ obeys the normalization $\iint d \theta d p f(\theta, p, t)=1 \forall t$; the magnetization is given by $M[f](t)=\iint d \theta d p f(\theta, p, t) e^{-i \theta}$.

The stationary states of Eq. (5) satisfy $\partial f_{\mathrm{s}} / \partial t=0$ so for these states, the divergence of the current density in phase space $\mathbf{J}=(\dot{\theta} f, \dot{p} f)$ is zero $[\nabla \cdot \mathbf{J}=0$, once the time derivative of Eq. (5) has been set to zero]. Let us focus on nonmagnetized stationary states, which correspond to $F\left[f_{\mathrm{s}}\right] \equiv 0$, so that any state $f_{0}(p)$ that is homogeneous in $\theta$ is a stationary solution of Eq. (5). Its linear stability is determined by considering the expansion $f(\theta, p, t)=f_{0}(p)+\delta f(\theta, p, t)$, with $\delta f$ 
being an eigenvector of the linearized dynamics whose norm satisfies $\|\delta f(\theta, p, t)\| \ll 1$, so that inserted in Eq. (5), one obtains to leading order the equation

$$
\frac{\partial \delta f}{\partial t}+p \frac{\partial \delta f}{\partial \theta}+F[\delta f](\theta, t) f_{0}^{\prime}(p)=0,
$$

where the prime denotes the derivative. Using the fact that $\delta f$ is $2 \pi$ periodic in $\theta$, we expand the perturbation $\delta f$ as $\delta f(\theta, p, t)=\sum_{k=-\infty}^{\infty} \widetilde{\delta f_{k}}(p) e^{i k \theta+\lambda t} ; \delta f$ being real implies that $\tilde{\delta f}_{-k}=\widetilde{\delta f}_{k}^{*}$. We then have $F[\delta f](\theta, t)=$ $-\pi \int d p^{\prime} \tilde{\delta f}_{k}\left(p^{\prime}\right) e^{i k \theta+\lambda t}\left(\delta_{k, 1}+\delta_{k,-1}\right)$. On substituting this expression in Eq. (6), we find that the Fourier coefficients $\widetilde{\delta f_{ \pm 1}}$ satisfy the equation $\widetilde{\delta f}{ }_{ \pm 1}(p)=\pi f_{0}^{\prime}(p) /(\lambda \pm$ ip) $\int d p^{\prime} \delta f_{ \pm 1}\left(p^{\prime}\right)$. On integrating both sides with respect to $p$ and noting that $\int d p \widetilde{\delta f}+1(p) \neq 0$, one gets the dispersion relation determining the stability parameter $\lambda$ :

$$
1=\pi \int d p \frac{f_{0}^{\prime}(p)}{\lambda \pm i p}
$$

On integrating by parts, the above equation gives the equality $\pi \int d p f_{0}(p) /(p \mp i \lambda)^{2}=i$ that can never be satisfied for $\lambda$ purely imaginary. We thus conclude that $\lambda$ is complex in general.

Let us first consider the so-called water-bag (WB) distribution, commonly used in studying long-range Hamiltonian systems [1] and inspired by plasma physics, where the particle momenta are uniformly distributed in a range $[-\Delta p ; \Delta p]$, with $\Delta p \geqslant 0$. The stability equation (7) translates into $\lambda^{2}=$ $i \pi-(\Delta p)^{2}$, which shows that if $\lambda$ solves the above equation, so does $-\lambda$, yet $\lambda$ cannot be pure imaginary. Thus, the stability equation will always admit a solution with positive real part, so that the WB distribution cannot be linearly stable under the dynamics (5).

We now consider a Gaussian state uniform in $\theta$ and Gaussian in $p: f_{0}(p)=1 /\left(2 \pi \sqrt{2 \pi \sigma^{2}}\right) \exp \left(-p^{2} /\left(2 \sigma^{2}\right)\right)$, with $\sigma>0$. Equation (7) gives

$$
\pm \frac{i}{\sqrt{\pi}\left(2 \sigma^{2}\right)^{3 / 2}}\left[\sqrt{2 \pi \sigma^{2}}-\pi \lambda e^{\lambda^{2} /\left(2 \sigma^{2}\right)} \operatorname{Erfc}\left(\lambda / \sqrt{2 \sigma^{2}}\right)\right]=1,
$$

where $\operatorname{Erfc}(x)$ is the complementary error function. Such Gaussian states appear to be stable under the Vlasov dynamics (5) for a width above $\sigma \approx 0.6$. Below this value, the distribution will evolve on a $N$-independent timescale toward a Vlasov-stable distribution. Let us remember that the condition (7) is quite general, so some non-Gaussian distributions may also be stable. For example, for a Lorentzian distribution $f_{0}(p)=\sigma / \pi\left(p^{2}+\sigma^{2}\right)$, the eigenvalues are $\lambda=$ $-\sigma \pm \sqrt{\pi / 2}(1+i)$, so that the distribution is stable provided its width obeys $\sigma>\sqrt{\pi / 2}$.

On the basis of the above discussion, and as confirmed numerically, the dynamics of a large system initially in a WB configuration relaxes to a Vlasov-stable stationary state on an $N$-independent timescale. Yet, since the system does not possess a proper equilibrium, its convergence to a Gaussian state (Boltzmann distribution if the system were Hamiltonian) is not granted.

To understand the evolution of the Vlasov-stable distribution for finite $N$, let us consider single particles: They are driven by the magnetization, which fluctuates around zero.
Using the definition of the magnetization, let us rewrite the single-particle dynamics by using Eq. (3) as

$$
\dot{p}_{j}=-\frac{1}{N}-\frac{\operatorname{Re}\left[\eta_{j}(t)\right]}{\sqrt{N}},
$$

where $\eta_{j}(t)=e^{i \theta_{j}(t)}(1 / \sqrt{N}) \sum_{m \neq j} e^{-i \theta_{m}(t)}$ is of order unity, and the factor $1 / N$ comes from the diagonal $j=m$ term in $M$. On timescales much smaller than $\sqrt{N}$, the resulting quasiballistic motion makes it possible to write that $\theta_{j}\left(t+t^{\prime}\right)-$ $\theta_{j}(t) \approx p_{j}(t) t^{\prime}$. Assuming that the particles have uncorrelated positions, we obtain that $\dot{P}=-|M|^{2}=-1 / N$ and

$$
\begin{aligned}
& \left\langle\eta_{j}(t) \eta_{j}^{*}\left(t+t^{\prime}\right)\right\rangle \\
& \approx e^{-i p_{j}(t) t^{\prime}} \frac{1}{N} \sum_{m \neq j} e^{i p_{m}(t) t^{\prime}}\left(1+\sum_{n \neq m} e^{i\left[\theta_{n}(t)-\theta_{m}(t)\right]}\right) \\
& \approx e^{-i p_{j}(t) t^{\prime}} \iint d \theta d p f_{t}(p) e^{i p t^{\prime}},
\end{aligned}
$$

where the double sum has been dropped in going from the

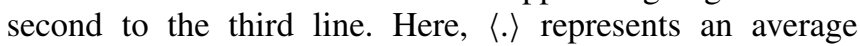
over configurations, and $f_{t}$ is the statistical average of the single-particle distribution $f$ at time $t$. For a Gaussian distribution $f_{t}=1 /(2 \pi) \exp \left[-(p-\bar{p})^{2} /\left(2 \sigma^{2}\right)\right] / \sqrt{2 \pi \sigma^{2}}$ centered around $\bar{p}$, one obtains

$$
\left\langle\eta_{j}(t) \eta_{j}^{*}\left(t+t^{\prime}\right)\right\rangle=\exp \left[-\frac{\sigma^{2} t^{2}}{2}-i\left(p_{j}-\bar{p}\right) t^{\prime}\right] .
$$

The phase term in Eq. (10) may be neglected since it varies little over the different values of $p_{j}$ (i.e., over the momentum distribution) for times smaller than the coherence time $t^{\prime}<1 / \sigma$. Consequently, for timescales larger than $1 / \sigma, \eta_{j}$ can effectively be considered as a white noise with $\langle\eta(t)\rangle=$ $0,\left\langle\eta(t) \eta\left(t+t^{\prime}\right)\right\rangle=D(\sigma) \delta\left(t^{\prime}\right)$, where the diffusion coefficient is obtained as

$$
D(\sigma)=\int\left\langle\eta_{j}(t) \eta_{j}\left(t+t^{\prime}\right)\right\rangle d t=\sqrt{\frac{\pi}{2}} \frac{1}{\sigma} .
$$

This behavior of the magnetization is illustrated in Fig. 2, where the autocorrelation in time of the magnetization is shown, presenting a clear decay in time over a scale that does not depend on the system size $N$. Moreover, this allows us to write a Fokker-Planck equation for the single-particle

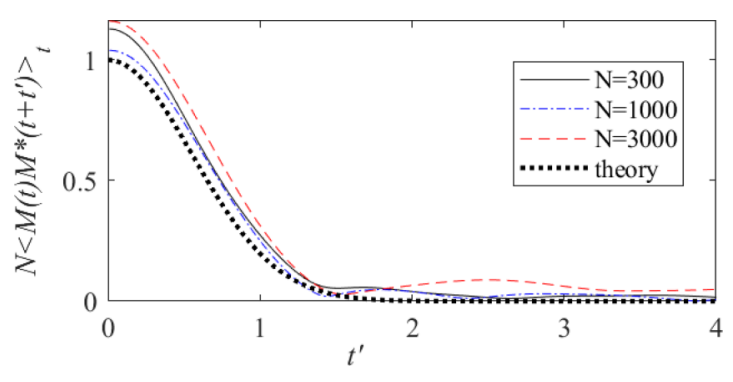

FIG. 2. Autocorrelation in time of the magnetization $M(t)$, computed after a time $100 \mathrm{~N}$, and over a time window $\Delta t=100$, starting from a WB initial state with $\Delta p=0.5$. The theory curve has been drawn using the measured width of the momentum distribution $\sigma \approx 1.8$. 
distribution $\mathcal{P}(p-P, t)$ centered around $P$ as

$$
\frac{\partial \mathcal{P}}{\partial t}=D(\sigma) \frac{\partial^{2} \mathcal{P}}{\partial p^{2}} .
$$

While this appears to be the equation of a Brownian motion, the dependence of the diffusion coefficient on the distribution makes it a nonlinear equation in $\mathcal{P}$, which does not possess an analytical solution [17]. Practically, as the distribution spreads in momentum, the diffusion coefficient decreases as the coherence time of $\eta(t)$ reduces, so that the diffusion actually slows down in time.

Before describing the above process in more detail, let us comment on the complete dynamical evolution starting from the initial WB state: After the initial transient that follows the relaxation from the WB state on a timescale that does not depend on the system size (a process often called violent relaxation [18]), the system reaches a state that statistically corresponds to a distribution which is a stationary and stable solution of the Vlasov equation. After that, the slow (quasistationary) relaxation occurs over timescales that grow linearly with the system size $N$, during which the system evolves toward a state Gaussian in momentum and homogeneous in $\theta$. This was checked numerically by monitoring the momenta of the distribution, which reached the values for a Gaussian distribution. The dynamical evolution is shown in Fig. 1.

The evolution of the distribution is then captured under the hypothesis that it is Gaussian at any time. Using the ansatz $\mathcal{P}(t)=1 /(2 \pi) \exp \left\{-(p-\bar{p})^{2} /\left[2 \sigma^{2}(t)\right]\right\} / \sqrt{2 \pi \sigma^{2}(t)}$ along with Eq. (11), one obtains $\sigma^{\prime} \sigma=D$, which yields

$$
\sigma^{3}(t)=\sigma^{3}(0)+3 \sqrt{\frac{\pi}{2}} t .
$$

This equation describes a subdiffusive behavior, where the distribution temperature $T \sim\left\langle(p-\bar{p})^{2}\right\rangle$ grows with time as $t^{2 / 3}$, instead of $t$ as for the standard Brownian motion, due to the fact that the spreading of the distribution in momentum continues concomitantly with a reduction of the diffusion coefficient (11). The validity of the Gaussian distribution ansatz is confirmed by the numerical observation of the subdiffusive behavior; see Fig. 3. This result bears strong similarities with those of Ref. [16], where anomalous diffusion was predicted for a similar infinite-range Hamiltonian system. In that case, the diffusion in the system also resulted from the weak coupling of many particles through a vanishing magnetization.

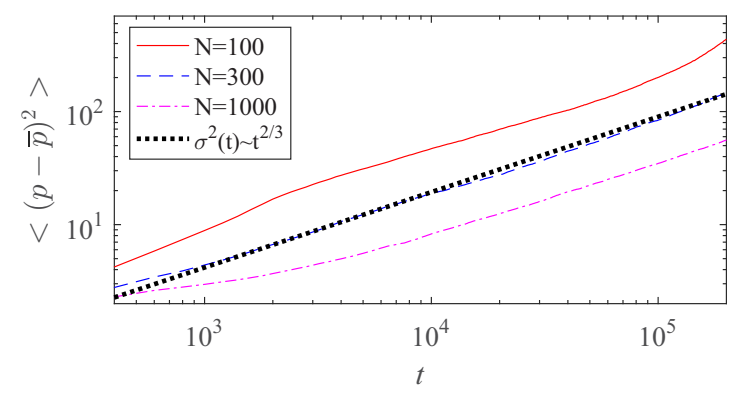

FIG. 3. Evolution of $\left\langle(p-\bar{p})^{2}\right\rangle$ for different system sizes and the subdiffusive behavior prediction, Eq. (13). The system is initially in a WB state with $\Delta p=0.5$.
In conclusion, we have shown that a non-Hamiltonian long-range system may present a slowdown of relaxation with the system size, similar to what is known for Hamiltonian systems under the name quasistationary states. The existence of a Vlasov equation for nonconservative systems driven by non-Hamiltonian two-body interaction (differently from, for example, systems with friction forces) allows for this approach to possess nonequilibrium stable stationary states, which translates into quasistationary states for the microscopic dynamics. The increase over time of the system temperature makes the interaction between the particles less effective. Because diffusion results from a mutual coupling between particles, the interplay between momentum diffusion and coupling leads to the observed subdiffusion. Other long-range systems with a similar coupling (i.e., particles interacting through a macroscopic magnetization, yet in a lowmagnetization phase) are expected to present similar behavior.

Note that preliminary simulations of the "hybrid" system (2) (with $\Delta<0$ and $\kappa>0$ ) suggest that, depending on the relative strength of each coupling term, the system will adopt either the behavior of the non-Hamiltonian term described in the present paper, or that of the Hamiltonian term studied in previous works $[14,19]$. Indeed, while it was not possible to identify a regime with quasistationary states with finite magnetization for the purely non-Hamiltonian case $(\Delta=0)$, the additional presence of the Hamiltonian term allows us to recover magnetized stable states. More precisely, when the coupling relative to the Hamiltonian part $2 g^{2} \Delta /\left(\kappa^{2}+\Delta^{2}\right)$ is larger (in absolute value) than the one relative to the nonHamiltonian part $2 g^{2} \kappa /\left(\kappa^{2}+\Delta^{2}\right)$, states appear that bear the characteristics of the low-energy quasistationary states of the Hamiltonian mean-field model $(\Delta<0$ and $\kappa=0)$. Whereas in the opposite case, an unmagnetized state appears, which presents characteristics of the non-Hamiltonian component (i.e., a drift of the average momentum on a timescale that grows linearly with the system size $N$ ). An illustration of this dichotomous behavior is presented in Fig. 4. This raises several interesting questions, such as the following: Does the

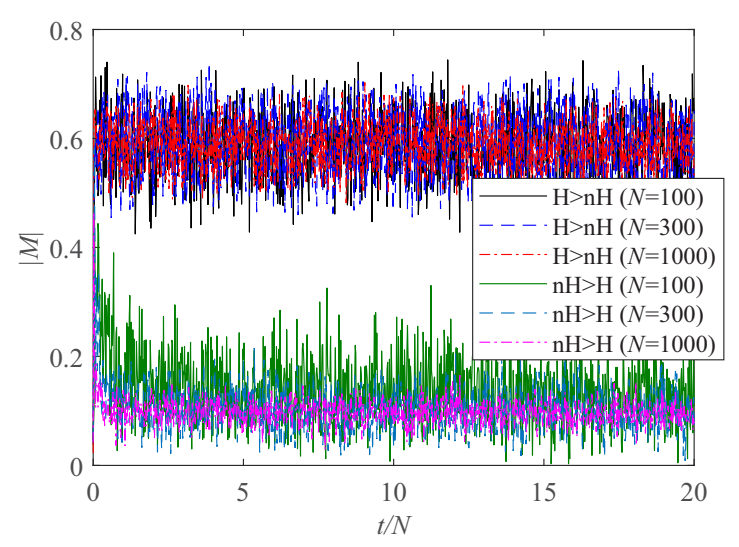

FIG. 4. Evolution of magnetization for a configuration where the Hamiltonian coupling is stronger than the nonsymmetric one ( $\Delta=-2 \kappa$ and $-2 g^{2} \kappa /\left(\kappa^{2}+\Delta^{2}\right)=1$, labeled "H $>\mathrm{nH}$ "), and for the opposite configuration $\left(\Delta=-\kappa / 2\right.$ and $-2 g^{2} \kappa /\left(\kappa^{2}+\Delta^{2}\right)=1$, labeled " $\mathrm{nH}>\mathrm{H}$ "), for different system sizes. The system is initially in a WB state with $\Delta p=0.5$. 
non-Hamiltonian term have an impact on the quasistationary lifetimes of the Hamiltonian component? What is the phase diagram for these two dynamics as a function of both coupling parameters $(\Delta / \kappa)$ and initial conditions? Indeed, quasistationary states are known to depend heavily on initial conditions [20], instead of temperature or energy for equilibrium phenomena. These intriguing questions are left for a future work.

Finally, let us comment that a particularly promising platform to investigate experimentally the aforementioned peculiar behavior is that of an ultracold cloud trapped in an optical cavity. In this case, the infinite-range interaction between the atoms mediated by the light is known to dominate the dynamics, and the leakage of the light through the cavity mirrors results in an overdamped dynamics [21]. These systems do not have a thermal equilibrium state, since the pump light keeps increasing the cloud momen- tum, driving the atoms farther from resonance. The fact that the momentum distribution is routinely tracked by time-offlight techniques makes these setups especially interesting for observing the predicted nonequilibrium anomalous diffusive behavior.

We thank Alessandro Campa for fruitful discussions and an anonymous referee for helpful suggestions. S.G. thanks Universidade Federal de São Carlos and the Centro de Pesquisa em Óptica e Fotônica (FAPESP 2013/07276-1), Brazil, for warm hospitality and financial support. R.B. is grateful for support from Fundaçãode Amparo à Pesquisa do Estado de São Paulo (FAPESP) (2014/01491-0 and 2015/50422-4). N.P. and R.B. participate in the EU H2020 ITN project ColOpt (No. 721465). The Titan X Pascal used for this research was donated by the NVIDIA Corporation.
[1] A. Campa, T. Dauxois, D. Fanelli, and S. Ruffo, Physics of Long-Range Interacting Systems (Oxford University Press, Oxford, UK, 2014).

[2] H. Touchette, Simple spin models with non-concave entropies, Am. J. Phys. 76, 26 (2008).

[3] H. Touchette, Equivalence and nonequivalence of ensembles: Thermodynamic, macrostate, and measure levels, J. Stat. Phys. 159, 987 (2015).

[4] Y. Y. Yamaguchi, Relaxation and diffusion in a globally coupled Hamiltonian system, Phys. Rev. E 68, 066210 (2003).

[5] T. M. R. Filho, A. E. Santana, M. A. Amato, and A. Figueiredo, Scaling of the dynamics of homogeneous states of onedimensional long-range interacting systems, Phys. Rev. E 90, 032133 (2014).

[6] Y. Y. Yamaguchi, J. Barré, F. Bouchet, T. Dauxois, and S. Ruffo, Stability criteria of the Vlasov equation and quasi-stationary states of the HMF model, Phys. A (Amsterdam, Neth.) 337, 36 (2004).

[7] S. Gupta and D. Mukamel, Slow Relaxation in Long-Range Interacting Systems with Stochastic Dynamics, Phys. Rev. Lett. 105, 040602 (2010).

[8] S. Gupta and D. Mukamel, Relaxation dynamics of stochastic long-range interacting systems, J. Stat. Mech.: Theory Exp. (2010) P08026.

[9] P.-H. Chavanis, F. Baldovin, and E. Orlandini, Noise-induced dynamical phase transitions in long-range systems, Phys. Rev. E 83, 040101(R) (2011).

[10] R. Bonifacio and F. Casagrande, Classical and quantum treatment of amplifier and superradiant free-electron laser dynamics, J. Opt. Soc. Am. B 2, 250 (1985).
[11] R. Bonifacio, G. R. M. Robb, and B. W. J. McNeil, Propagation, cavity, and Doppler-broadening effects in the collective atomic recoil laser, Phys. Rev. A 56, 912 (1997).

[12] M. Kac, G. E. Uhlenbeck, and P. C. Hemmer, On the van der Waals theory of the vapor-liquid equilibrium. I. Discussion of a one-dimensional model, J. Math. Phys. 4, 216 (1963).

[13] R. Bonifacio, L. De Salvo Souza, P. Pierini, and N. Piovella, The superradiant regime of a FEL: Analytical and numerical results, Nucl. Instrum. Methods Phys. Res. A 296, 358 (1990).

[14] M. Antoni and S. Ruffo, Clustering and relaxation in Hamiltonian long-range dynamics, Phys. Rev. E 52, 2361 (1995).

[15] S. Gupta and S. Ruffo, The world of long-range interactions: A bird's eye view, Int. J. Mod. Phys. A 32, 1741018 (2017).

[16] F. Bouchet and T. Dauxois, Prediction of anomalous diffusion and algebraic relaxations for long-range interacting systems, using classical statistical mechanics, Phys. Rev. E 72, 045103 (2005).

[17] T. D. Frank, Nonlinear Fokker-Planck Equations: Fundamentals and Applications (Springer, Berlin, 2005).

[18] D. Lynden-Bell, Statistical mechanics of violent relaxation in stellar systems, Mon. Notices Royal Astron. Soc. 136, 101 (1967).

[19] A. Campa, T. Dauxois, and S. Ruffo, Statistical mechanics and dynamics of solvable models with long-range interactions, Phys. Rep. 480, 57 (2009).

[20] A. Antoniazzi, D. Fanelli, S. Ruffo, and Y. Y. Yamaguchi, Nonequilibrium Tricritical Point in a System with Long-Range Interactions, Phys. Rev. Lett. 99, 040601 (2007).

[21] H. Ritsch, P. Domokos, F. Brennecke, and T. Esslinger, Cold atoms in cavity-generated dynamical optical potentials, Rev. Mod. Phys. 85, 553 (2013). 